\title{
Lesiones del himen en la determinación médico legal de la integridad sexual *
}

\author{
José Pacheco $D^{1}$, Felio Palomino ${ }^{1}$, Nancy De La Cruz ${ }^{1}$
}

Resumen

Palabras clave
Objetivo: Determinar las lesiones himeneales y sus variantes morfológicas. Materiales y Métodos: Se realizó un estudio descriptivo prospectivo de julio a agosto del 2005. Resultados: Hubo 284 exámenes por denuncias de VS; 80,3\% correspondió a niños y adolescentes. Los principales hallazgos fueron himen dilatable (36,9\%), himen íntegro (34,8\%), desgarros antiguos (13.6\%), himen dilatable con lesiones recientes (4,5\%), desgarros recientes (1,5\%), entre otros. Conclusiones: El himen muy elástico que presenta un orificio amplio, crea dificultad en el reconocimiento de lesiones recientes. Determinar un himen 'complaciente' o 'dilatable' no niega categóricamente la introducción del pene u otro miembro del cuerpo u objeto. La Medicina Legal enseña las variantes himeneales y sus principales lesiones para que todo examinador tenga el suficiente criterio técnico y científico al momento de acreditar sus hallazgos ante una autoridad judicial o fiscal.

Himen; violación; medicina legal; jurisprudencia.
Hymen injuries in medico-legal evaluation of sexual integrity

\section{Abstract}

Objective: To determine hymenal injuries and morphologic variants. Materials and Methods: Prospective and descriptive study done during July and August 2005. Results: There were 284 examinations for sexual violation denunciations; $80,3 \%$ corresponded to children and adolescents. The main findings were: dilatable hymen (36,9\%), integral hymen (34,8\%), old ruptures (13.6\%), dilatable hymen with recent injuries (4,5\%), recent ruptures (1,5\%), among others. Conclusions: The elastic hymen with ample orifice originates difficulty in the recognition of recent injuries. An 'accommodating'or 'dilatable' hymen does not categorically determine the introduction of the penis or other member of the body or object. Legal Medicine teaches on hymenal variants and main injuries so that any examiner has enough technical and scientific knowledge at the time of crediting his findings to judicial or fiscal authorities.

* El resumen del presente trabajo fue seleccionado como uno de los mejores durante las VII Jornadas Científicas San Fernandinas y publicado en: An Fac Med. 2005;66 Suppl 1:S40.

1 Instituto de Patología. Facultad de Medicina, UNMSM. Lima, Perú.
Keywords: Hymen; rape; forensic medicine; jurisprudence.

\section{INTRODUCCIÓN}

Recientemente, la legislación ha modificado la definición de violación sexual (VS) en nuestro país ya que el Código Penal del año 1991 limitaba las interpretaciones legales de este delito $\left({ }^{1}\right)$. En la actualidad, el Código Penal vigente ya no relaciona a la VS como un hecho realizado con excesiva fuerza física por parte del violador, ni tampoco, con la introducción del pene a la vagina como único mecanismo de producción.

Desde junio del año 2004, la Ley No 28251 modificó el Artículo $170^{\circ}$ del Código Penal relacionado a la VS. Esta modificatoria establece que se comete VS cuando una persona "con violencia o grave amenaza obliga a otra persona a tener acceso carnal por vía vaginal, anal o bucal o realiza actos análogos introduciendo objetos o partes del cuerpo por alguna de las dos primeras vías" $\left({ }^{2}\right)$. Este concepto ha generado la necesidad del perfeccionamiento en 
el diagnóstico forense de las lesiones en el himen y en la región mucocutánea del ano.

Investigaciones científicas nacionales han reportado las diferentes características del himen, así como la importancia de presentar estadísticas reales y confiables para efectos de educación sexual y para la aplicación en el ámbito jurídico $\left(^{3}\right)$. Asimismo, se ha publicado la importancia de conocer los criterios diagnósticos útiles en la definición de una VS. Anteriormente, un estudio nacional propuso que sólo se aseguraba la existencia de VS si anatómicamente se evidenciaba un desgarro himeneal reciente o antiguo $\left({ }^{4}\right)$.

Estudios internacionales también han resaltado lo importante que resulta determinar las lesiones himeneales en menores de edad, ya que existen casos de confusión entre las ocasionadas sexualmente y las producidas por accidente al introducirse juguetes, manejar bicicletas, entre otros traumatismos $\left(^{5-8}\right)$. Otras investigaciones sugieren la realización de constantes descripciones del himen para compararlas con las diferentes edades en la población $\left({ }^{9}\right)$.

Según lo expuesto, las nuevas definiciones jurídicas implican la urgente necesidad de difundir conocimientos médico legales relacionados a la determinación de integridad sexual, tanto en sus concepciones legales como forenses. Los conocimientos deben ser trasmitidos a todo aquel profesional de salud que examina y examinará a las personas denunciantes de este delito. Todo médico general y demás médicos de especialidades no forenses, entre otros profesionales, deberán estar capacitados en la identificación de una lesión himeneal, la cual tendrá definitivamente una trascendencia legal.

La presente investigación tendrá por objetivo determinar las lesiones del himen más frecuentes en personas que han denunciado una supuesta VS. Además, se expondrán los parámetros anatómicos, forenses y jurídicos para ser utilizados por los profesionales de salud en sus futuras evaluaciones clínicas con implicancias médico legales.

\section{MATERIALES Y MÉTODOS}

Estudio descriptivo prospectivo realizado en una División Médico Legal ubicada en Lima durante los meses de julio y agosto de 2005. Se seleccionó todas las pericias médico legales de integridad sexual, las cuales habían sido solicitadas por Fiscalías, Juzgados o dependencias de la Policía Nacional.

Se solicitó la autorización y el consentimiento a las personas examinadas para la respectiva toma fotográfica de las lesiones himeneales. Por motivos académicos y éticos, se omitió la identificación de las personas evaluadas.

Se consideró como variables de estudio al total de personas denunciantes de VS que acudieron a la evaluación médico legal. Se determinó y describió las características del himen así como sus principales lesiones. Las lesiones himeneales fueron clasificadas según la existencia de desgarros recientes (dentro de los 10 días de producido el hecho) o de desgarros antiguos (más de 10 días) $\left({ }^{10}\right)$.

En las personas que refirieron el antecedente de menarquia, se determinó con mayor precisión las características elásticas del himen. Los hímenes con elasticidad aumentada fueron establecidos por presentar un diámetro transhimeneal mayor a $30 \mathrm{~mm}$ (hímenes dilatables o complacientes), ya que cifras inferiores a esa medida son consideradas normales $\left({ }^{11}\right)$.

En las menores de edad que no presentan la menarquia (prepuberales), se consideran los diámetros transhimeneales de 4 a $15 \mathrm{~mm}$ como valores normales. Estos datos métricos fueron obtenidos de investigaciones realizadas en 1839 menores, según lo reportado en la literatura médica $\left.{ }^{(12}\right)$. Otros estudios han utilizado una medida de $10 \mathrm{~mm}$ como diámetro máximo normal $\left({ }^{13-17}\right)$. Para el presente estudio, se consideró como valor máximo (en ausencia de 
penetración) al diámetro transhimeneal igual a $10,5 \mathrm{~mm}\left({ }^{18}\right)$.

De igual forma, se brindó una gran importancia a los desgarros himeneales antiguos que presentaban lesiones contusas recientes, como tumefacción, equimosis, entre otras. Toda evaluación siempre fue realizada en presencia de un personal femenino (auxiliar administrativo, policía custodio, madre o familiar).

Se excluyó a las personas que se negaron a la realización del examen médico legal. Los datos fueron analizados con la ayuda del programa Microsoft Excel 2000.

\section{RESULTADOS}

Durante los meses de julio y agosto del año 2005, se realizó un total de 4398 reconocimientos médico legales, siendo un 6,5\% (284/4398) el porcentaje reportado como exámenes por delitos contra la libertad sexual (DCLS). El resto de reconocimientos lo conformaban los exámenes por lesiones $(36,5 \%)$, psicológicos forenses $(23 \%)$, violencia familiar $(16,3 \%)$, edad aproximada $(6,6 \%)$, entre otros.

La mayoría de personas que acudieron al examen médico legal por DCLS fue niños y adolescentes (menores de 18 años de edad) (Figura 1).

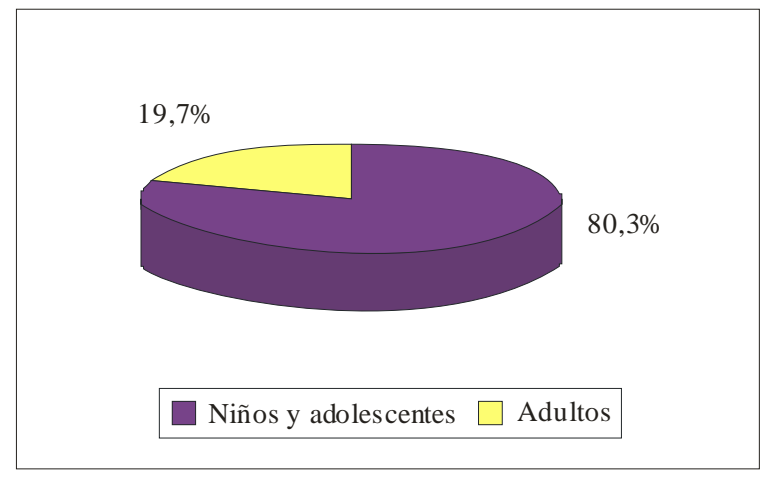

Figura 1. Personas evaluadas por delitos contra la libertad sexual (DCLS) según mayoría de edad.
De las 284 evaluaciones médico legales realizadas con el fin de determinar la integridad sexual en personas que denunciaron una VS, se evidenció que $79,2 \%(225 / 284)$ y $20,8 \%$ (59/ 284) correspondieron al sexo femenino y masculino, respectivamente.

Las personas de sexo femenino fueron clasificadas según grupo etáreo, observándose una mayor frecuencia en el rango de 6 a 17 años de edad (Tabla 1).

Tabla 1. Personas de sexo femenino que denunciaron una violación sexual, según edad.

\begin{tabular}{lrr}
\hline Rango de edad en años & $\mathrm{n}$ & \multicolumn{1}{c}{$\%$} \\
\hline 0 a 5 & 11 & 3,9 \\
6 a 12 & 52 & 18,3 \\
13 a 17 & 115 & 40,5 \\
18 a 23 & 17 & 6,0 \\
24 a más & 30 & 10,6 \\
Total & 225 & 100,0 \\
\hline
\end{tabular}

De las 225 personas de sexo femenino, 27 no autorizaron la realización del examen médico legal, siendo 198 evaluaciones, la población total del presente estudio.

Se evidenció que $76,8 \%(152 / 198)$ de los casos presentaron una ausencia de lesiones himeneales, siendo clasificados de acuerdo a su morfología (Figura 2). De éstos, 34,8\% (69/

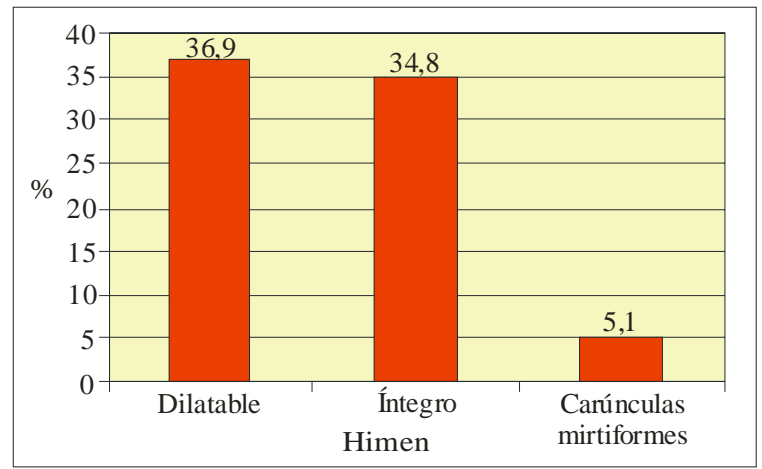

Figura 2. Clasificación de los hímenes sin lesiones durante la determinación médico legal de integridad sexual. 

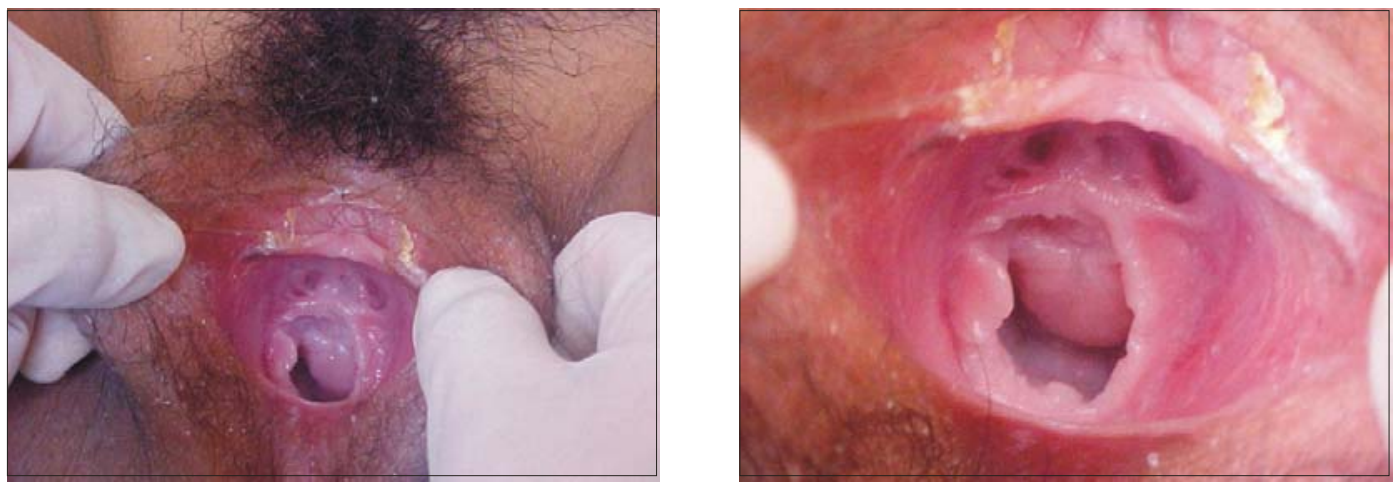

Foto 3. Himen dilatable. Nótese el aumento del diámetro transhimeneal al realizar la exposición.

198) presentó un himen íntegro (borde interno que delimita el orificio sin lesiones). Asimismo, $36,9 \%(73 / 198)$ fue calificado como himen dilatable (o complaciente) por presentar una elasticidad aumentada y un orificio amplio, tal como se explicó anteriormente (Figuras 3 y 4). Un $5,1 \%(10 / 198)$ fue relacionado a la presencia de carúnculas mirtiformes (rezagos del himen debido a un parto anterior).

De igual forma, se determinó que 23,2\% (46/ 198) de los casos presentó lesiones himeneales, siendo clasificados de acuerdo a sus características macroscópicas en lesiones recientes (dentro de los 10 días de sucedido el hecho) y antiguas (más de 10 días) (Figuras 5, $6,7,8$ y 9). Los desgarros himeneales antiguos representaron el 13,6\% (27/198), seguidos en frecuencia de los hímenes dilatables con signos de lesiones recientes, con 4,5\% (9/198).

\section{DISCUSIÓN}

El reconocimiento o examen médico legal es un procedimiento médico de gran implicancia en el sistema de administración de justicia, siendo solicitado por las autoridades competentes (fiscalías, juzgados, Policía Nacional y, de acuerdo a las ultimas normas jurídicas, por la Demuna (Defensoría Municipal del Niño y el Adolescente)) $\left({ }^{19,20}\right)$. Esta evaluación la puede realizar cualquier médico en ausencia del especialista en Medicina Legal, según lo
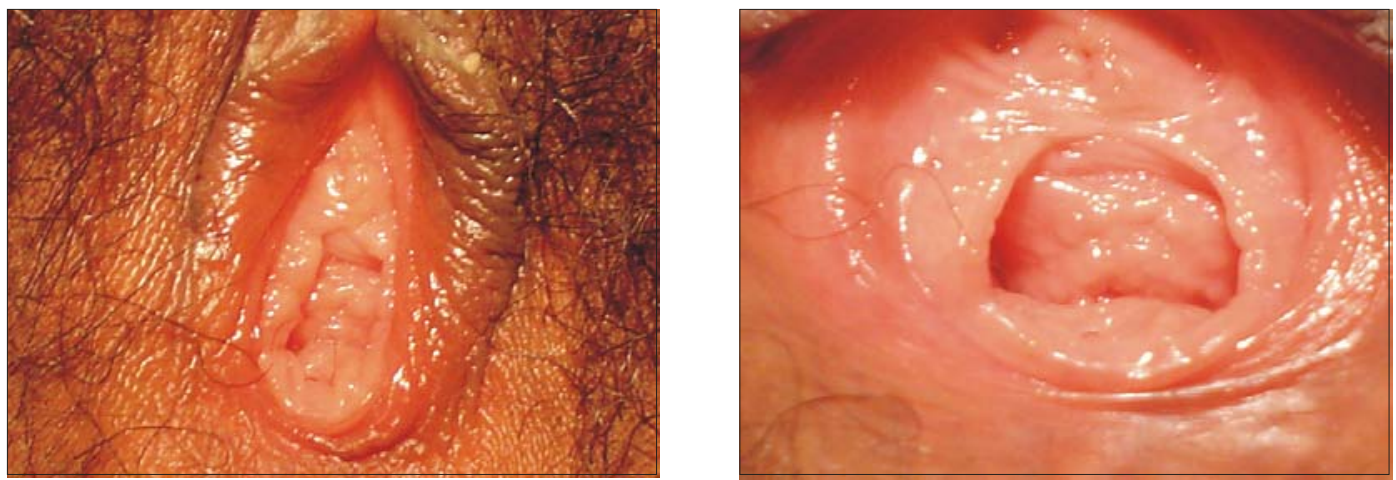

Foto 4. Himen dilatable. Se evidencia un orificio amplio a la exposición y el borde libre interno no presenta lesiones. 


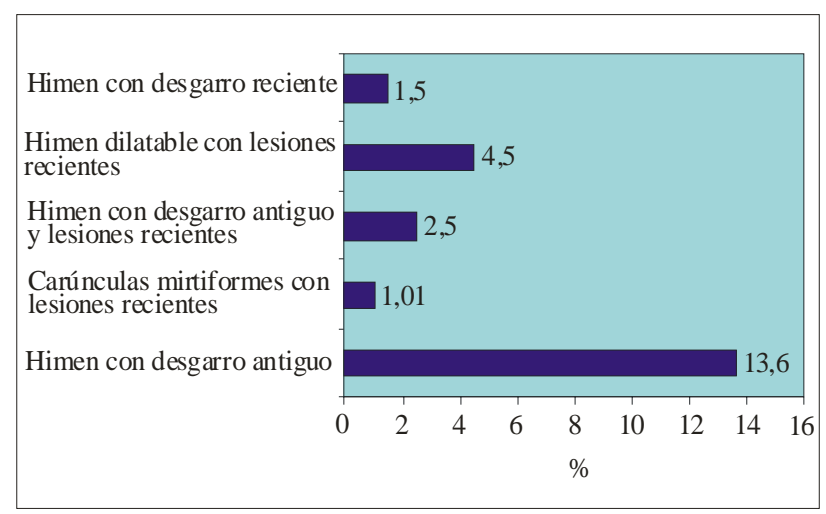

Figura 5. Clasificación de los hímenes con lesiones durante la determinación médico legal de integridad sexual.

establecido en el Código de Procedimientos Penales ${ }^{(21)}$. El resultado de esta evaluación será emitido en un documento llamado Certificado médico legal, el cual deberá ser redactado técnica y científicamente de acuerdo al examen solicitado.

Ante una supuesta violación sexual, la autoridad competente designará al profesional responsable de la evaluación clínica de la persona denunciante. Tal como lo establece nuestra normatividad, el juez instructor deberá nombrar de preferencia a especialistas donde los hubiere $y$, en ausencia de éstos, a quienes se encuentren sirviendo al Estado (médicos serumistas). A falta de profesionales, se nombrará a una persona de reconocida honorabilidad y competencia en la materia $\left({ }^{21}\right)$. Por lo tanto, cualquier profesional de salud será un candidato útil para colaborar con la administración de justicia.

Para la investigación del hecho que constituye el delito o para la identificación de los culpables, se empleará todos los medios científicos y técnicos que fuesen posibles $\left({ }^{22}\right)$. Por ello, en una víctima de violación sexual, el profesional examinador deberá obtener toda evidencia que se relacione con este delito, tal como vellos púbicos, manchas de semen y muestras de contenido vaginal y/o anal, entre otros. Las investigaciones han determinado la utilidad del recojo de evidencias de interés forense hasta 44 horas después del acto sexual $\left({ }^{23}\right)$. Otros autores también han propuesto recientes métodos científicamente comprobados que pueden ayudar a la visualización de las lesiones himeneales tales como el uso de un catéter Foley insuflado $\left({ }^{24}\right)$.

Según nuestra selección de casos, consideramos muy importante el resaltar que las evaluaciones fueron realizadas en mujeres que autorizaron la pericia. Un examen médico legal siempre será practicado, previo consentimiento de la víctima, exclusivamente por el médico encargado del servicio, con la asistencia de un
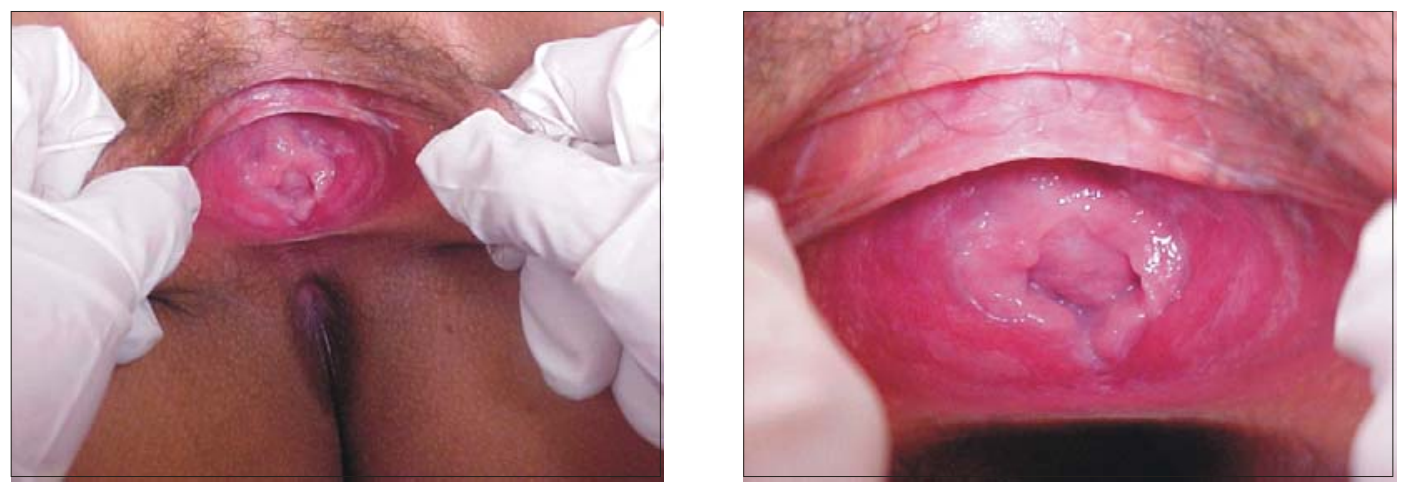

Foto 6. Himen con desgarro completo antiguo. Nótese la ruptura a horas VI según el cuadrante de un reloj. Los bordes de la lesión están cubiertos por un tejido cicatricial (color blanco nacarado). 

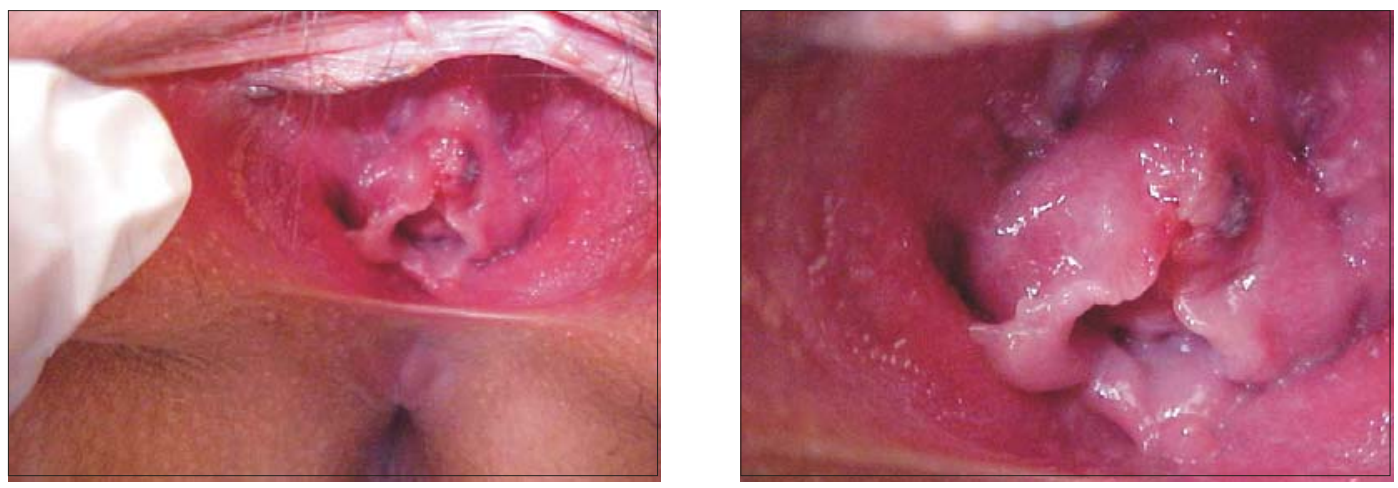

Foto 7. Himen con desgarro incompleto reciente. Nótese la ruptura a horas XII según el cuadrante de un reloj. Los bordes de la lesión presentan signos de inflamación aguda (tumefacción y sangrado).

profesional auxiliar. Asimismo, se permitirá la presencia de otras personas, previo consentimiento de la víctima ${ }^{(25)}$.

Los hallazgos himeneales descritos durante la nuestra evaluación se relacionaron con signos traumáticos a nivel genital. Siendo el pene, los dedos u otros objetos duros de superficie roma, agentes clasificados como contundentes, se observará lesiones denominadas contusas $\left({ }^{26}\right)$. Así pues, las lesiones del himen relacionadas a un abuso sexual serán identificadas y evidenciadas como desgarros o laceraciones, equimosis y tumefacciones del borde himeneal $\left({ }^{27}\right)$.
La mayoría de personas que denunció una violación sexual estuvo en el rango de 6 a 17 años de edad. Nuestros datos también se relacionan con estudios publicados internacionalmente, ya que en las últimas dos décadas el número de menores de edad víctimas de abuso sexual se ha incrementado dramáticamente $\left({ }^{28}\right)$.

Se ha establecido que los hímenes pierden su morfología anatómica después de producirse un parto vaginal. Nuestros hallazgos muestran que es posible determinar lesiones recientes sobre los signos de partos anteriores, rezagos
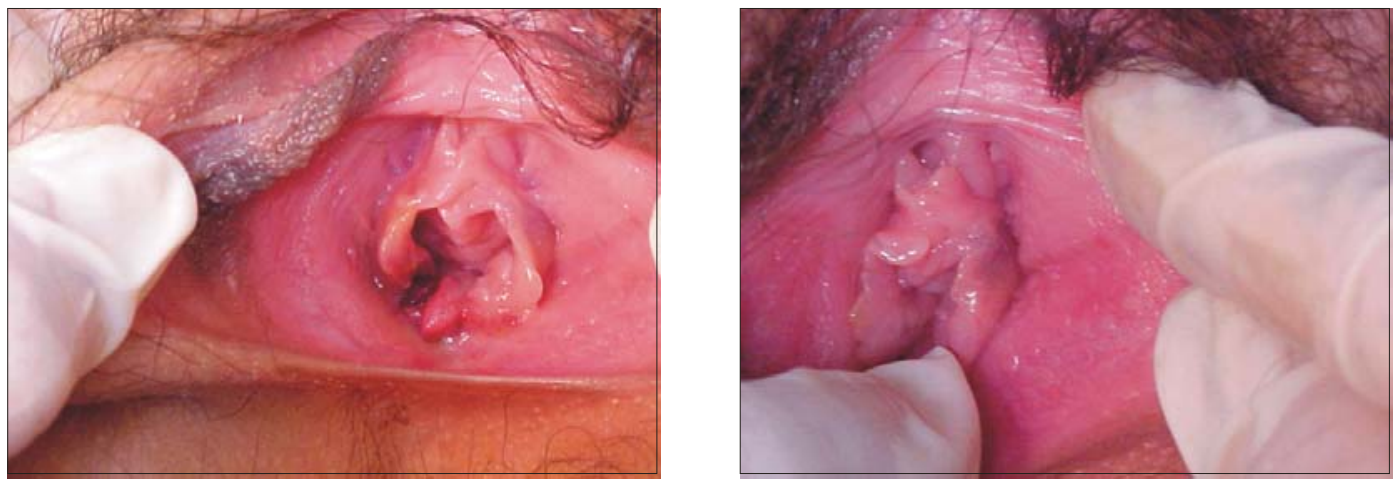

Foto 8. Himen con desgarro completo reciente. Nótese la ruptura a horas VII según el cuadrante de un reloj. Los bordes de la lesión presentan signos de sangrado. A la derecha: Equimosis en borde de inserción himeneal en horas III. 
himeneales que son denominados carúnculas mirtiformes. Otros autores han descrito también estos hallazgos en adolescentes gestantes, quienes habían sido victimas de un abuso sexual $\left({ }^{29}\right)$.

Un porcentaje significativo fue vinculado con los denominados hímenes 'complacientes'. Cuando el himen tiene la propiedad de ser muy elástico y presentar un orificio muy amplio, crea una dificultad al profesional que no tiene experiencia en reconocer la existencia de lesiones recientes. Determinar un himen 'complaciente' o 'dilatable' no niega categóricamente la introducción del pene u otro miembro del cuerpo u objeto. Como antecedente, cabe aquí destacar que, anteriormente, el himen dilatable era catalogado si es que el orificio himeneal permitía el paso de dos dedos y si, después de retirarlos, el diámetro volvía a su tamaño normal $\left({ }^{4}\right)$.

Esta determinación de hímenes dilatables no deberá ser realizado por descarte. Actualmente, muchos profesionales experimentan un temor cuando no están capacitados en identificar lesiones himeneales y plantean erróneamente el diagnóstico de himen 'complaciente' con el fin de evitar futuras ratificaciones y confrontaciones legales. Para ello, la Medicina Legal brinda los medios técnicos y científicos necesarios para esta correcta determinación, los cuales son renovados constantemente según lo comunicado a nivel internacional $\left({ }^{30}\right)$.

Hemos mencionado que los certificados médico legales que determinan las lesiones himeneales son muy útiles en la tipificación del delito de violación sexual. Sin embargo, se ha señalado que muchas veces estos documentos médicos, aún siendo positivos en la descripción de lesiones himeneales, no guardan una asociación con el resultado final del proceso legal trayendo, como consecuencia el archivo del caso $\left({ }^{31}\right)$.

Por lo tanto, la Medicina Legal, ciencia que está científicamente avanzando a pasos agigantados, enseña las diferentes variantes himeneales y sus lesiones para que todo examinador tenga el suficiente criterio técnico y científico al momento de acreditar sus hallazgos ante una autoridad judicial o fiscal.

\section{REFERENCIAS BIBLIOGRÁFICAS}

1. Código Penal. Delitos contra la libertad sexual. Art. $170^{\circ}$ : Violación sexual; Perú, 1991. Disponible en: http:// www.cajpe.org.pe/rij/bases/legisla/peru/pecodpen.htm

2. Ley $\mathrm{N}^{\circ} 28251$. Modificación del Art. $170^{\circ}$ del Código Penal; Perú, Junio 2004. Disponible en: http://www.cajpe.org.pe/ rij/bases/legisla/peru/28251.pdf

3. Berrios MR, Rodríguez PJ, Salazar GS, Tapara QJ. Delitos contra la libertad sexual: Frecuencia de himen complaciente en la ciudad de Arequipa 2002. Revista Científica del Instituto de Medicina Legal. Marzo 2005;1. Disponible en: http://www.mpfn.gob.pe/iml/revista.php\#

4. Latoche FE, Latoche RC. Estudio anatómico del himen y su repercusión en medicina legal. Med Leg Costa Rica. 1999;16(1-2):2-7.

5. Boos SC. Accidental hymenal injury mimicking sexual trauma. Pediatrics. 199;103(6):1287-90.

6. Pokorny SF, Pokorny WJ, Kramer W. Acute genital injury in the prepubertal girl. Am J Obstet Gynecol. 1992;166:1461-6.

7. Dowd MD, Fitzmaurice L, Knapp JF, Mooney D. The interpretation of urogenital findings in children with straddle injuries. J Pediatr Surg. 1994;29:7-10.

8. Boos SC, Rosas AJ, Boyle C, McCann J. Anogenital injuries in child pedestrians run over by low-speed motor vehicles: four cases with findings that mimic child sexual abuse. Pediatrics. 2003;112(1 Pt 1):e77-84.

9. Abdullah S. The hymen morphology in normal newborn Saudi girls. Ann Saudi Med. 2001;21(3-4):188-9.

10. Vargas Alvarado E. Medicina Forense y Deontología Médica. $2^{\text {da }}$ ed. México: Editorial Trillas; 1999.

11. Adams JA, Botash AS, Kellogg N. Differences in hymenal morphology between adolescent girls with and without a history of consensual sexual intercourse. Arch Pediatr Adolesc Med. 2004;158(3):280-5.

12. Perry AP. Genital findings in prepubertal girls evaluated for sexual abuse: A different perspective on hymenal measurements. Arch Fam Med. 1999;8:403-6.

13. Cantwell HB. Vaginal inspection as it relates to child sexual abuse in girls under thirteen. Child Abuse Neglect. 1983;7:171-6.

14. Huffman JW, Dewhurst CJ, Capraro MF. The Gynecology of Childhood and Adolescence. $2^{\text {da }}$ ed. Philadelphia, Pa: WB Saunders Co.; 1981.

15. Muram D. Child sexual abuse: genital tract findings in prepubertal girls: the unaided medical examination. Am J Obstet Gynecol. 1989;160:328-33. 

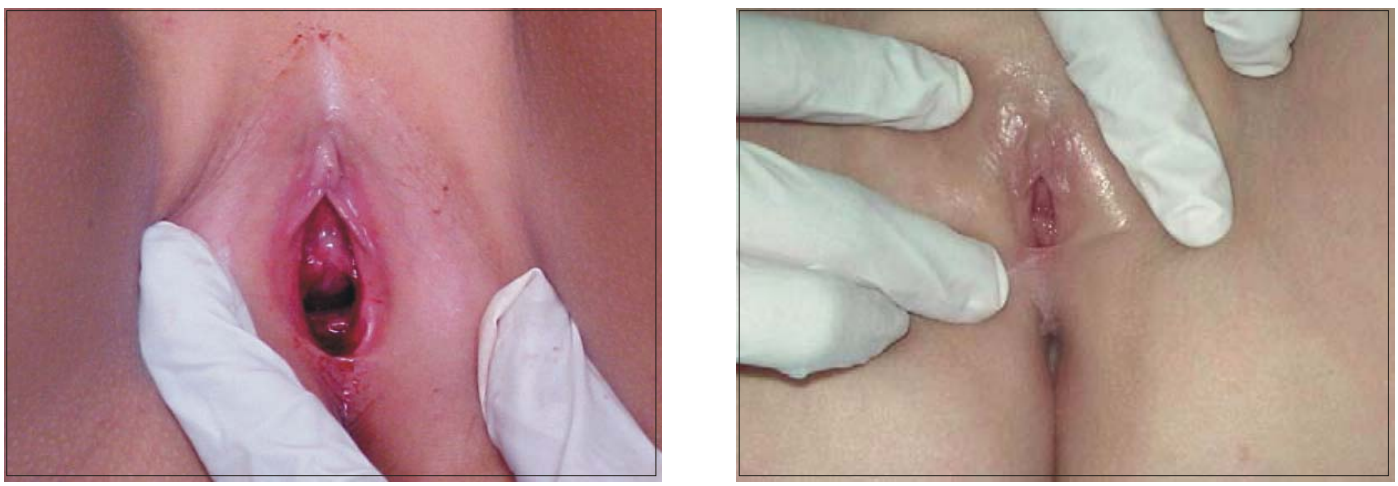

Foto 9. Menor de 9 años de edad con un desgarro himeneal reciente (abundante signos de sangrado). A la derecha: Características normales del introito pre puberal.

16. Paradise JE. Predictive accuracy and the diagnosis of child abuse: a big issue about a little tissue. Child Abuse Neglect. 1989;13:169-76

17. White ST, Ingram DL, Lyna PR. Vaginal introital diameter in the evaluation of sexual abuse. Child Abuse Neglect. 1989; 123:217-24.

18. Berenson AB, Chacko MR, Wiemann CM, Mishaw CO, Friedrich WN, Grady JJ. Use of hymenal measurements in the diagnosis of previous penetration. Pediatrics. 2002;109(2):228-35.

19. Reglamento del Servicio de Defensoría del Niño y Adolescente. Art. $16^{\circ}$, inciso c; Perú, 1999.

20. Código de los Niños y Adolescentes. Articulo $27^{\circ}$; Perú, 1992.

21. Código de Procedimientos Penales. Nombramiento de Peritos. Art. $161^{\circ}$ vigente; Perú, 1939.

22. Código de Procedimientos Penales. Uso de medios científicos. Art. $194^{\circ}$ vigente; Perú, 1939.

23. Christian CW, Lavelle JM, De Jong AR, Loiselle J, Brenner L, Joffe M. Forensic evidence findings in prepubertal victims of sexual assault. Pediatrics. 2000;106(1):100-4.

24. Jones JS, Dunnuck C, Rossman L, Wynn BN, Genco M. Adolescent Foley catheter technique for visualizing hymenal injuries in adolescent sexual assault. Acad Emerg Med. 2003;10(9):1001-4.

25 . Ley $\mathrm{N}^{\circ} 27115$. Ley que establece la acción penal pública en los delitos contra la libertad sexual. Art. $3^{\circ}$ inciso 2; Perú, Mayo 1999. Disponible en: http://www.cajpe.org.pe/rij/ bases/legisla/peru/pen46.htm

26. Gisbert Calabuig JA. Medicina legal y toxicología. $6^{\mathrm{a}}$ ed. Madrid: Editorial Masson; 2004.
27. Adams J. Evolution of a classification scale: Appendix. Child Maltreatment. 2001;6(1):34-5. Disponible en: http:// www.gwmedical.com/sexualassault/tableerratum.pdf

28. Lung CT, Daro D. Current trends in child abuse reporting and fatalities: The results of the 1995 Annual Fifty State Survey. Chicago, Ill: The National Committee to Prevent Child Abuse; 1996. p. 9.

29. Kellogg ND, Menard SW, Santos A. Genital anatomy in pregnant adolescents: "normal" does not mean "nothing happened". Pediatrics. 2004;113(1 Pt 1):e67-9.

30. Ingram DM, Everett VD, Ingram DL. The relationship between the transverse hymenal orifice diameter by the separation technique and other possible markers of sexual abuse. Child Abuse Negl. 2001;25(8):1109-20.

31. McGregor MJ, Le G, Marion SA, Wiebe E. Examination for sexual assault: Is the documentation of physical injury associated with the laying of charges? A retrospective cohort study. Canadian Med Assoc J. 1999;160(11):1565-9.

Manuscrito recibido el 20 de octubre de 2005 y aceptado para publicación el 30 octubre de 2005.

Correspondencia: Dr. José Luis Pacheco De La Cruz Hospital Nacional Arzobispo Loayza Instituto de Patologia, UNMSM.

Av. Alfonso Ugarte 848

Lima 1, Perú

Correo-e: jpachecodelacruz@yahoo.es jlpd60@hotmail.com 